Please do not remove this page

RMIT

UNIVERSITY

\title{
Gravitational Effect on the Formation of Surface Nanodroplets
}

Yu, Haitao; Lu, Ziyang; Lohse, Detlef; Zhang, Xuehua

https://researchrepository.rmit.edu.au/esploro/outputs/9921862691001341/filesAndLinks?institution=61RMIT_INST\&index=null

Yu, H., Lu, Z., Lohse, D., \& Zhang, X. (2015). Gravitational Effect on the Formation of Surface Nanodroplets. Langmuir, 31(46), 12628-12634. https://doi.org/10.1021/acs.langmuir.5b03464

Document Version: Accepted Manuscript

Published Version: https://doi.org/10.1021/acs.langmuir.5b03464

Repository homepage: https://researchrepository.rmit.edu.au

(c) 2015 American Chemical Society

Downloaded On 2023/04/27 01:26:49 +1000

Please do not remove this page 
Thank you for downloading this document from the RMIT Research Repository.

The RMIT Research Repository is an open access database showcasing the research outputs of RMIT University researchers.

RMIT Research Repository: http://researchbank.rmit.edu.au/

\section{Citation:}

Yu, H, Lu, Z, Lohse, D and Zhang, X 2015, 'Gravitational Effect on the Formation of Surface Nanodroplets', Langmuir, vol. 31, no. 46, pp. 12628-12634.

See this record in the RMIT Research Repository at:

https://researchbank.rmit.edu.au/view/rmit:34182

Version: Accepted Manuscript

Copyright Statement: (c) 2015 American Chemical Society

Link to Published Version:

Description

http://dx.doi.org/10.1021/acs.langmuir.5b03464 


\title{
Gravitational effect on the formation of surface nanodroplets
}

\author{
Haitao Yu, ${ }^{\dagger}$ Ziyang Lu, ${ }^{\dagger}$ Detlef Lohse, ${ }^{\ddagger}$ and Xuehua Zhang ${ }^{*} \dagger$
}

Soft Matter \& Interfaces Group, School of Civil, Environmental and Chemical Engineering, RMIT University, Melbourne, VIC 3001, Australia, Physics of Fluids group, Department of

Science and Engineering, Mesa+ Institute, and J. M. Burgers Centre for Fluid Dynamics, University of Twente, P.O. Box 217, 7500 AE Enschede, The Netherlands, and Max Planck Institute for Dynamics and Self-Organization, 37077 Goettingen, Germany

E-mail: xuehua.zhang@rmit.edu.au

\begin{abstract}
Nanoscale droplets at a solid-liquid interface are of high relevance for many fundamental phenomena and applied processes. The solvent exchange process is a simple approach to produce e.g. oil nanodroplets over a large surface area on a substrate, by exchange oil-saturated ethanol by oil-saturated water, which has a lower oil solubility than ethanol. In this process, the size of the nanodroplets is closely related to the flow conditions. To achieve control of the droplet size, it is essential to fully understand the nucleation and growth of nanodroplets under different flow conditions. In this work, we investigate the gravitational effect on the droplet formation by the solvent exchange.
\end{abstract}

\footnotetext{
*To whom correspondence should be addressed

†Soft Matter \& Interfaces Group, School of Civil, Environmental and Chemical Engineering, RMIT University, Melbourne, VIC 3001, Australia

${ }^{\ddagger}$ Physics of Fluids group, Department of Science and Engineering, Mesa + Institute, and J. M. Burgers Centre for Fluid Dynamics, University of Twente, P.O. Box 217, 7500 AE Enschede, The Netherlands

`Max Planck Institute for Dynamics and Self-Organization, 37077 Goettingen, Germany
} 
We compared the droplet size as the substrate was placed on the upper or lower wall in a horizontal fluid channel or on the sides of a vertical channel with an upward or downward flow. We found significant difference in the droplet size for the three substrate positions in a wide channel with height $h=0.21 \mathrm{~mm}$. The difference of droplet size was eliminated in a narrow channel with height $h=0.07 \mathrm{~mm}$. The relevant dimensional control parameter for the occurrence of the gravitational effects is the Archimedes number Ar and these two heights correspond to $A r=10$ and $A r=0.35$, respectively. The gravitational effects lead to a non-symmetric parabolic profile of the mixing front, with the velocity maximum being off-center and thus with different distances $\alpha(A r) h$ and $(1-\alpha(A r)) h$ to the lower and upper wall, respectively. The ratio of the total droplet volume on the lower and upper wall is theoretically found to be $(\alpha(A r) /(1-\alpha(A r)))^{3}$. This study thus improves our understanding of the mechanism of the solvent exchange process, providing guidelines for tailoring the volume of surface nanodroplets.

\section{Introduction}

Surface nanodroplets refer to the droplets at a solid-liquid interface with at least one dimension less than $1 \mu \mathrm{m}$. Those nanodroplets draw great interest from both fundamental studies and broad applications. ${ }^{1-4}$ Such systems possess fascinating stability and colloidal properties. $^{5-7}$ They act as miniaturised reactors in formulation industry, ${ }^{8,9}$ microcontainers for high throughput screening and analysis, ${ }^{10-13}$ and adjustable lenses in high-resolution nearfield imaging technique. ${ }^{14}$ The solvent exchange is a simple approach to form nanodroplets (or bubbles) at a solid-liquid interface. ${ }^{15,16}$ This approach is versatile for the type of droplets (oils, or water) and planar or non-planar substrates. ${ }^{17}$ The droplets can be controlled to be as small as several attoliters and be arranged on well-defined locations. ${ }^{18}$ The basic requirements for the solvent exchange are: (a) two miscible solvents (the first: solution A; the second: solution B), (b) the droplet liquid with a higher solubility in solution A than in solution B, and (c) a substrate with an appropriate wettability. ${ }^{1}$ 
To arbitrarily tailor the volume of surface nanodroplets, it is essential to fully understand the principle of the solvent exchange. The latest work by us and our co-workers has reported the primary mechanism that drives the heterogeneous nucleation and growth of droplets in this process: A pulse of oil oversaturation is created at the mixing front between solution $\mathrm{A}$ and $\mathrm{B}$, the intensity and duration of which dominate the droplet nucleation and growth. ${ }^{16}$ The theoretical analysis showed that the volume of the nanodroplets increases with the Peclet number $(\mathrm{Pe})$ of the flow as $\propto \mathrm{Pe}^{3 / 4}$, in good agreement with the experimental results. ${ }^{16}$ Gravitational effects were noticed in high channels where the convection facilitates the mixing and the formation of larger and more heterogenous droplets. In this work, we try to understand whether the gravity plays a role in a channel that is already narrow enough to eliminate convectional effects. Very interestingly, we found that the volume of surface nanodroplets in a horizontal channel is still subject to gravitational effects till the channel height is less than $70 \mu \mathrm{m}$. The insight from this work will be valuable for the effective design in scale-up of nanodroplet formation.

\section{Experimental section}

Solutions and Substrate: The monomer and initiator was 1,6-hexanediol di-acrylate (HDODA) (80\%, Sigma-Aldrich) and 2-hydroxy-2-methylpropiophenone (97\%, Sigma-Aldrich) respectively. A stock solution of monomer precursors, served as oil-rich phase, was prepared by mixing 10 volume of monomer and 1 volume of initiator. Solution A was obtained after $4 \mathrm{~mL}$ of the stock solution was dissolved into $100 \mathrm{~mL} 50$ vol\% ethanol aqueous solution. Solution B was HDODA-saturated water.

Silicon coated with a monolayer of octadecyltrichlorosiliane (OTS-Si) was prepared by

following the protocol reported in previous work, ${ }^{18}$ and was used as the substrate in all experiments. Before use, OTS-Si substrate was sonicated in ethanol for $10 \mathrm{mins}$ and dried under a nitrogen stream. It was further cleaned by a $\mathrm{CO}_{2}$ snow injector (30 Gunjet Spraying 
Systems CO. Wheaton, IL USA.) to remove any aggregates on the surface. ${ }^{19}$

Solvent exchange: The top and side view of the fluid cell and solvent exchange process are sketched in Fig. 1a. During the solvent exchange process, solution A with high oil solubility filled the fluid cell first, and then was displaced by solution B with low oil solubility. The solvent exchange speed is controlled at a constant flow rate of solution B by using a syringe pump. In order to identify the gravity effect in the solvent exchange process, four sets (Fig. 1b) at different tilting angles $\left(0,90^{\circ}, 180^{\circ}\right.$ and $\left.270^{\circ}\right)$ were designed and tested. After the completion of the solvent exchange, the oil nanodroplets on the substrate were illuminated with an UV lamp $(365 \mathrm{~nm}, 20 \mathrm{~W})$ for 15 minutes to order to solidify the nanolenses by photopolymerization. Finally, the substrate was washed with ethanol and dried under a gentle nitrogen stream. After this, the polymerized surface droplets on the substrate was characterised by a reflection-mode optical microscopy and tapping-mode atomic force microscopy (AFM).

Flow conditions: In our experiment, the width $(w)$ and height $(h)$ of the fluid cell are 15 $\mathrm{mm}$ and $0.21 \mathrm{~mm}$, respectively. The flow conditions are listed in Table 1 , where $\bar{U}$ is the mean flow velocity, the flow rate $Q=h w \bar{U}$, the Reynolds number of flow $R e=\bar{U} h / \nu=Q /(w \nu)$ and Peclet number $P e=\bar{U} h / D$. Here $\nu$ is the viscosity $\left(\nu=10^{-6} \mathrm{~m}^{2} / \mathrm{s}\right)$ and $D$ is the diffusivity $\left(D \approx 1.0 \times 10^{-9} \mathrm{~m}^{2} / \mathrm{s}\right) \cdot{ }^{20,21}$ All of our experiments are in the regime of laminar flow, i.e. $R e<1$.

Table 1: Flow rates during the solvent exchange. The dimensions of the flow channel were constant in all experiments.

\begin{tabular}{cccc}
\hline $\mathrm{Q}(\mu \mathrm{L} / \mathrm{min})$ & $\mathrm{Re}$ & $\mathrm{Pe}$ & $\mathrm{U}(\mathrm{mm} / \mathrm{s})$ \\
\hline 100 & 0.04 & 111 & 0.53 \\
200 & 0.08 & 222 & 1.06 \\
400 & 0.17 & 444 & 2.12 \\
800 & 0.34 & 889 & 4.23 \\
1600 & 0.67 & 1778 & 8.47 \\
\hline
\end{tabular}


(a) Top view and side view
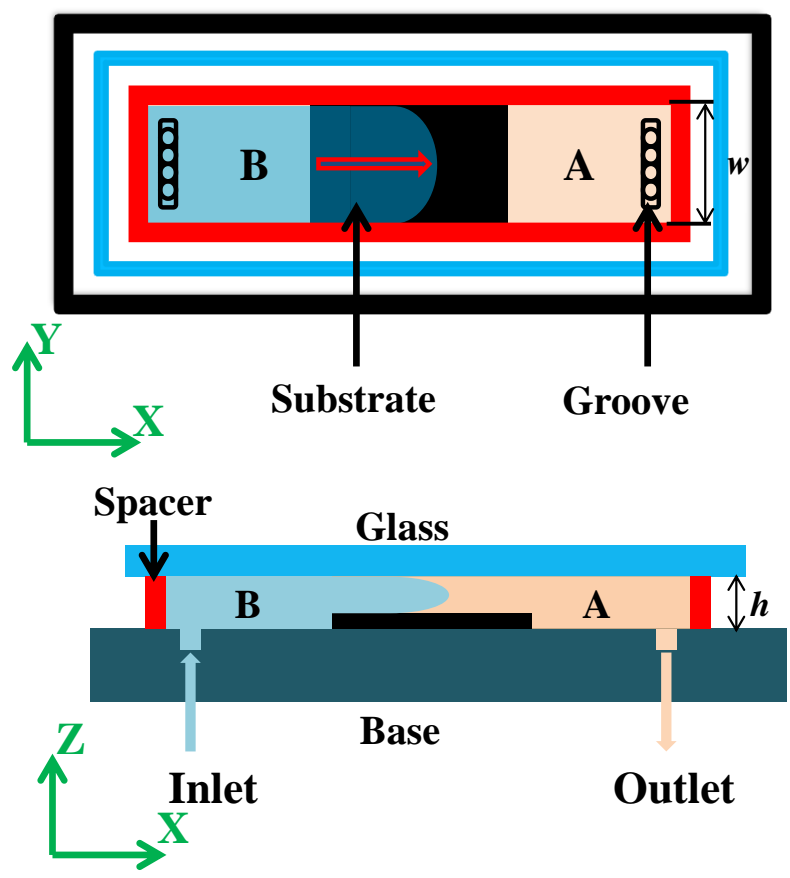

(b) Experimental setup
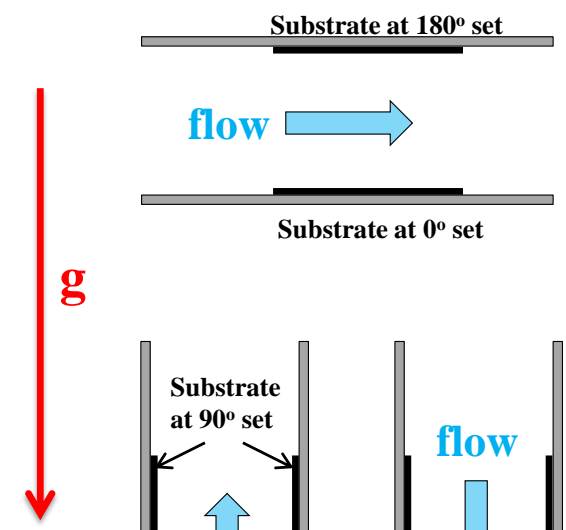

g

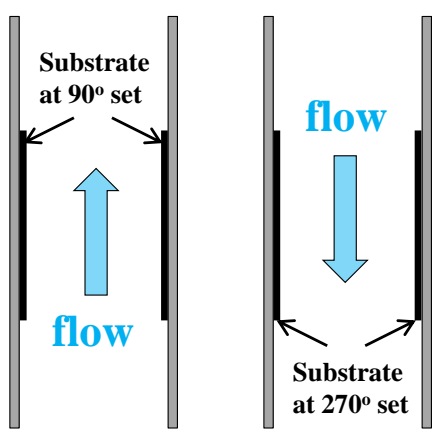

Figure 1: Illustration of the solvent exchange process and the positioning of the fluid channel. (a) The fluid cell consists of a base, a spacer and a glass top. The hydrophobic substrate was fixed on the base. (b) The four configurations $\left(0^{\circ}, 90^{\circ}, 180^{\circ}\right.$ and $\left.270^{\circ}\right)$ that are examined in this manuscript. When the fluid cell was placed horizontally, the flow was always parallel to the substrate placed either on the bottom $\left(0^{\circ}\right.$ flow $)$ or on the top $\left(180^{\circ}\right.$ flow). When the fluid cell and the substrate were placed vertically, the flow was either pumped upwards $\left(90^{\circ}\right.$ flow) or downwards (270 flow). 


\section{Results}

The morphology of droplets produced on horizontal and vertical substrates were characterized to examine the gravity effect. Figs. 2a and b show a representative three-dimensional view and the plot of the droplet height versus the lateral size. The shape of all the droplets are spherical caps with a height $(\mathrm{H})$ of $50 \mathrm{~nm}$ to $1.2 \mu \mathrm{m}$, a lateral diameter (L) of $2-40 \mu \mathrm{m}$. The ratio between the droplet height and the lateral diameter is approximately 0.03 , corresponding to a contact angle around $6^{\circ}$. So the droplet morphology is same for different tilting angles and not influenced by gravity, which is expected as the droplet size is so small that the capillarity force dominates.

Optical images in Fig. 2c show the droplet size as the substrate was positioned at different angles. The droplets are significantly larger on the substrate that was faced down than that faced up toward the flow at the same rate of $100 \mu \mathrm{L} / \mathrm{min}$. Moreover, the probability distribution function $(\mathrm{PDF})$ in Fig. 2d illustrates that the distribution of droplet size can be varied dramatically by simply turning around the fluid channel with an angle of $0^{\circ}, 90^{\circ}$, $180^{\circ}$ to $270^{\circ}$.

We further examined the gravity effects on the droplet size by varying the flow rate from $200 \mu \mathrm{L} / \mathrm{min}$ to $1600 \mu \mathrm{L} / \mathrm{min}$ at each fixed angle. As shown in Fig. 3, for a given flow rate the droplet size always follow the same order on the substrate of $0^{\circ}<90^{\circ}<180^{\circ}$. For low flow rates of less than $800 \mu \mathrm{L} / \mathrm{min}$, an downward flow on a $270^{\circ}$ substrate produced much larger droplets than an upward flow on a $90^{\circ}$ substrate. However, this difference diminished as the flow rate increases to $1600 \mu \mathrm{L} / \mathrm{min}$, and the droplet size became the same from both upward and downward flow.

The droplet volume per unit surface area was calculated according to the lateral diameter and the droplet contact angle of $6^{\circ}$ resulting from Fig. 2b. The analysis shows that the volume of the droplets increases with the flow rate. For a fixed angle of $0^{\circ}, 90^{\circ}$ or $180^{\circ}$,

the droplet size increases with the Peclet number of the flow as $V o l \propto h^{3} \mathrm{Pe}^{3 / 4}$, as shown in Fig. 4. This is consistent with the recent report on the droplet formation under controlled 

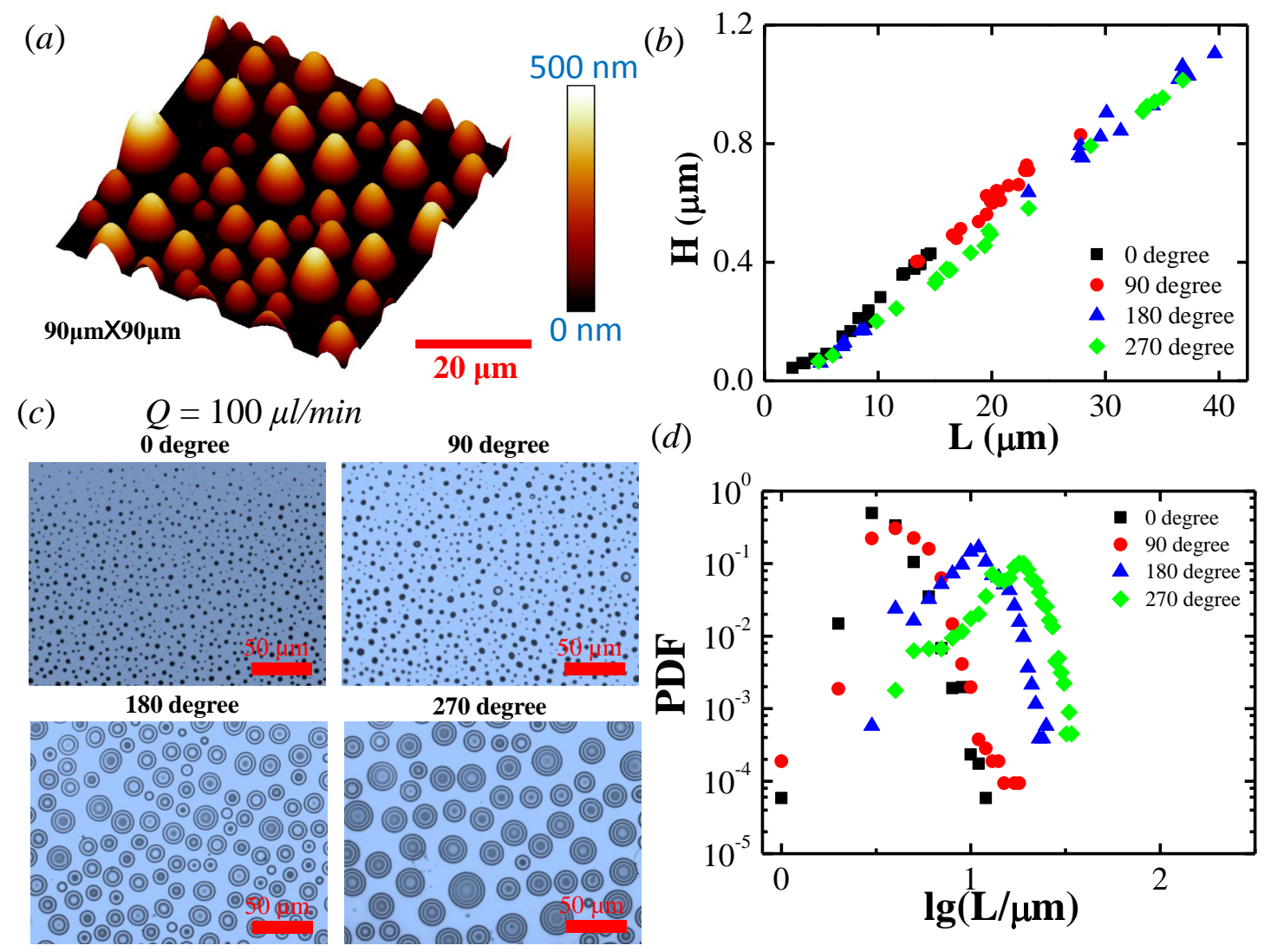

Figure 2: The morphologic features, optical image and size distribution of the surface droplets formed in the solvent exchange process. (a) Representative 3D AFM image of the polymerised surface droplets. (b) Height $\mathrm{H}$ versus lateral diameter $\mathrm{L}$ of the surface droplets. The slope is certified to be nearly independent of the tilting angle. So the contact angle of surface droplet is independent of the tilting angle as well. $(c)$ Reflection-mode optical images of the ploymerized surface droplets produced at the flow rate $100 \mu \mathrm{L} / \mathrm{min}$ of four tilting angles $(0$, 90, 180 and 270 degree). (d) PDF versus L (in $\mu \mathrm{m}$ ) on a logarithmic scale. 


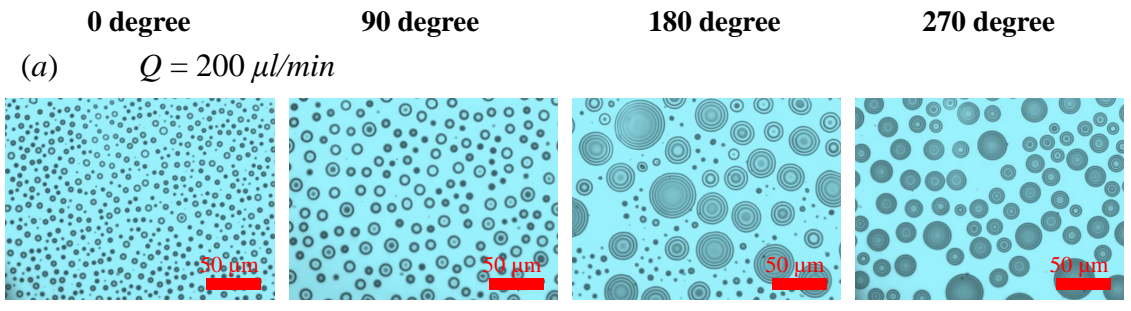

(b) $\quad Q=400 \mu l / m i n$

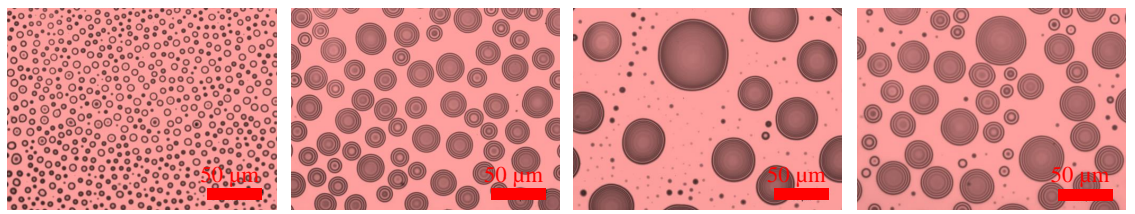

(c) $Q=800 \mu \mathrm{l} / \mathrm{min}$
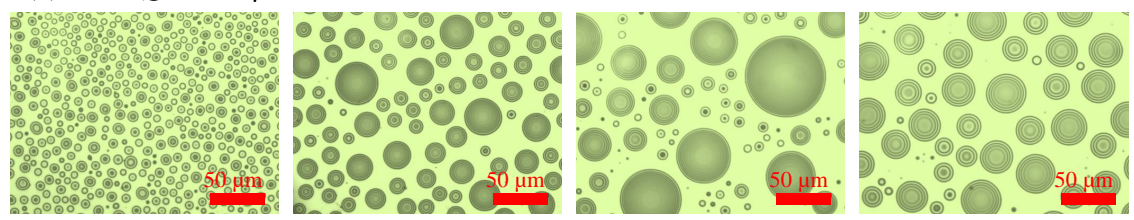

(d) $\quad Q=1600 \mu \mathrm{l} / \mathrm{min}$
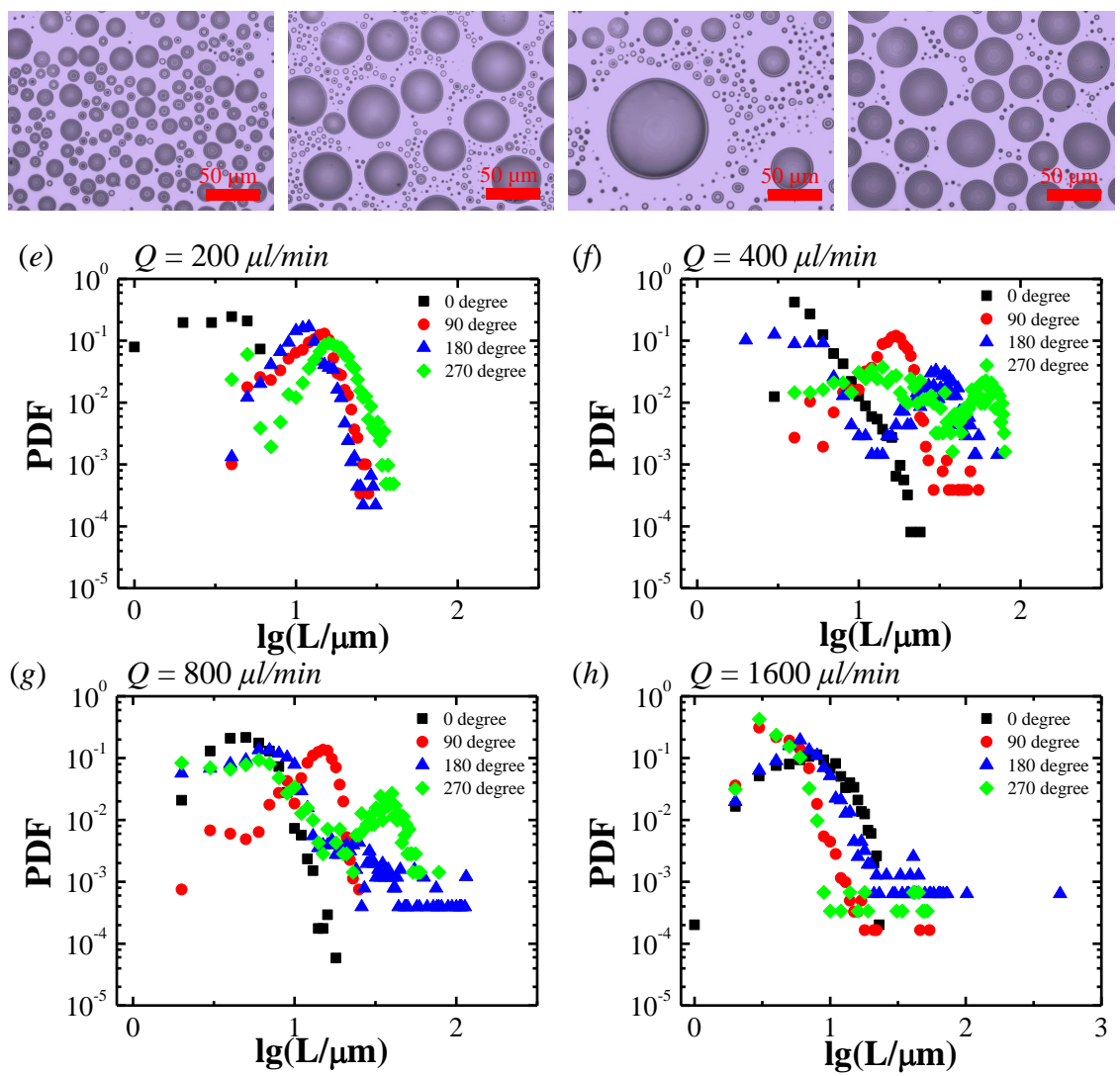

Figure 3: Optical images and PDF of four substrate positions at various flow rates. ( $a$ d) More optical images of four positioning angles at different flow rates: $200 \mu \mathrm{L} / \mathrm{min}$, $400 \mathrm{\mu L} / \mathrm{min}, 800 \mathrm{\mu L} / \mathrm{min}$ and $1600 \mu \mathrm{L} / \mathrm{min}$. (e-h) The corresponding results of PDF versus $\mathrm{L}(\mu \mathrm{m})$ on a logarithmic scale to optical images $(a-d)$. 
flow conditions. Our results show that the scaling law $\left(V o l \propto h^{3} \mathrm{Pe}^{3 / 4}\right)$ is valid not only on the horizontal substrate at the angle of $0^{\circ},{ }^{16}$ but also on the substrate at the angle of $90^{\circ}$ or $180^{\circ}$.

(a)

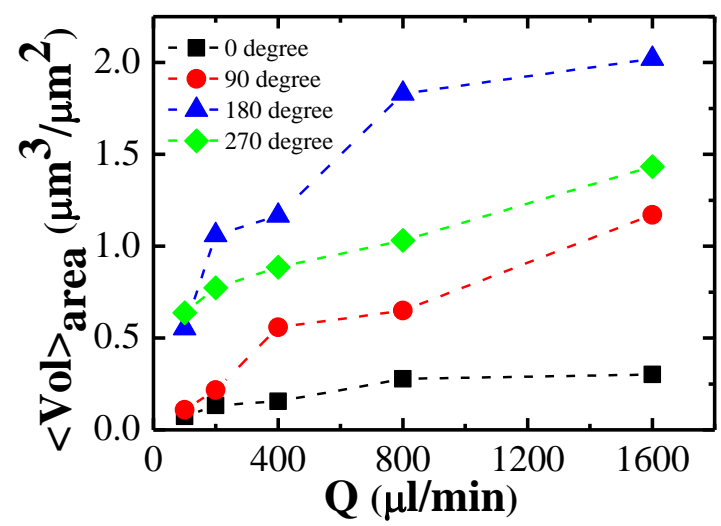

(b)

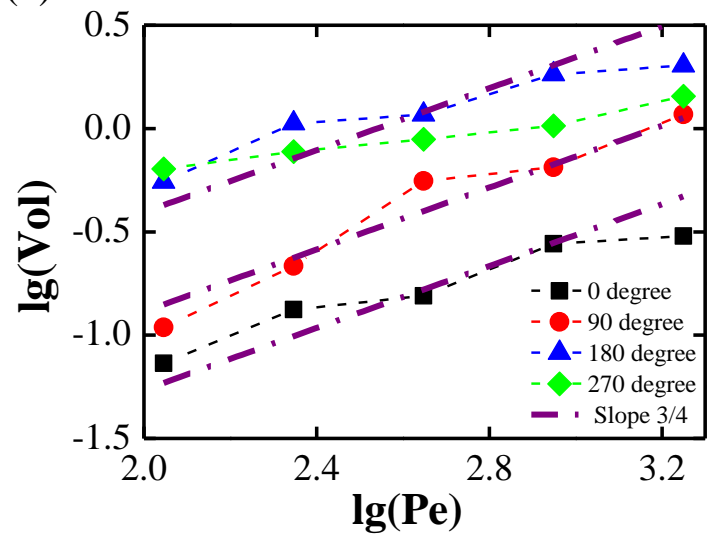

Figure 4: Size of surface droplets formed at different tilting angles and flow rates under the channel height $0.21 \mathrm{~mm}$. (a) Averaged volume per $\mu^{2}$ of surface droplets as a function of various flow rates of four tilting angles. (b) Averaged volume of surface droplets versus the Peclet number on a log-log plot. The dashed lines show the slope of $3 / 4$, corresponding to the scaling law $V o l \propto h^{3} \mathrm{Pe}^{3 / 4}$.

After having shown the Peclet number scaling relations of the droplet volume for various orientations as derived in ref. 16, we would now like to address the prefactor. Therefore we fit the data for the volume $(V o l)$ in Fig. 4b with the scaling law $V o l=f(\theta) h^{3} \mathrm{Pe}^{3 / 4}$, with an orientation dependent prefactor $f(\theta)$. The ratio of these prefactors between the upper $\left(\theta=180^{\circ}\right)$, the lower $\left(\theta=0^{\circ}\right)$, and the vertical $\left(\theta=90^{\circ}\right)$ wall is $6.3: 1: 2.5$. We will explain soon that this ratio is exactly due to the gravitational effect.

\section{Discussion}

We will now explain the gravity effects on the droplet size. The sketch in Fig. 5a illustrates a perfect parabolic profile of the moving front where the mixing between two solutions occurs, providing the oversaturated oil for the formation and growth of surface droplets. The volume of droplets increases with the Peclet number of the flow as $V o l \propto h^{3} \mathrm{Pe}^{3 / 4} .{ }^{16}$ However, an 
ideal parabolic profile of the moving front of the solvent exchange is only the case in absence of gravity effect. In general, however, one solution is lighter than the other one, with a density difference $\Delta \rho$. In our case the lighter solution A (with density $0.9 \mathrm{~kg} / \mathrm{m}^{3}$ ) is displaced by a flow of denser solution B (with density $\rho=1.0 \mathrm{~kg} / \mathrm{m}^{3}$ ), i.e., $\Delta \rho=0.1 \mathrm{~kg} / \mathrm{m}^{3}$. As the channel is laid flat, the profile of moving front may not be symmetric any more, due to the density difference between solution A and B. The profile of the moving front is shifted downwards, and consequently the position of the maximal flow velocity is lower than $h / 2$ of the channel.

The crucial control parameter controlling these gravity effects is the Archimedes number $^{22-24}$

$$
A r=\frac{g h^{3}}{\nu^{2}} \frac{\Delta \rho}{\rho}
$$

where $g=9.81 \mathrm{~m} / \mathrm{s}^{2}$ is the gravitational acceleration and $\nu=10^{-6} \mathrm{~m}^{2} / \mathrm{s}$ is the kinematic viscosity. For large $A r \gg 1$ (i.e. larger density difference or channel height) gravity plays a prominent role, whereas for small $A r \ll 1$ (i.e. smaller density difference or channel height) the gravity effect can be neglected. In our experiments $h=0.21 \mathrm{~mm}, \Delta \rho / \rho=0.1$, and $\nu=10^{-6} \mathrm{~m}^{2} / \mathrm{s}$, and we obtain $A r \approx 10$. So the gravity indeed plays a substantial role on the shape of the front profile. Later we will perform experiments in a $h=0.07 \mathrm{~mm}$ channel, resulting in $A r \approx 0.35$. Indeed, in that case we will not find effects of the orientation of the channel, i.e., no gravity effects.

How does the effect of gravity, which becomes dominant for $A r \geq 1$, qualitatively work? The maximum of the flow will no longer be in the middle of the (horizontal) channel, but be shifted slightly down, due to the denser water (solution B) as compared to ethanol (solution A). Therefore the oil-rich ethanol is pushed upwards, where it will nucleate larger droplets on the upper plate than on the bottom plate.

To further quantify the gravity effects, we define the position of the velocity peak $\left(V_{\max }\right)$ in a horizontal fluid channel. $V_{\max }$ is at $\alpha h$ with $\alpha$ from 0 to 0.5 . In the case $V_{\max }$ at the half channel resulted from a symmetrical parabolic profile (e.g., for $A r \rightarrow 0), \alpha$ is 0.5 . When $V_{\max }$ is on the bottom wall (e.g., for $\left.A r \rightarrow \infty\right) \alpha$ is 0 , and concurrently it is 1 for the upper 
wall. Ideally, one should calculate $\alpha(A r)$ for the solvent exchange process. However, the detailed flow pattern will be complicated and time dependent; we will therefore leave $\alpha$ as free parameter to be determined from our data.

One crucial idea of our theory of ref. 16 was to assume typical time scale for the nanodroplet growth as the diffusive time scale $\tau \sim h^{2} / D$. When considering gravitational effects, this assumptions needs an extension, namely we use the position of the velocity peak $\alpha h$ for the estimate of $\tau$. The relevant length scale is the distance between velocity maximum and wall, which determines the diffusion time $\tau$ for the droplet growth, $\tau \sim(\alpha h)^{2} / D$ where $\mathrm{D}$ is the diffusion constant. The consequence from a squeezed or stretched profile is the difference of diffusion time for the droplet growth on the upper and lower walls, which is $\alpha h$ for the latter and $(1-\alpha) h$ for the former.

By doing so, with the same reasoning and derivation as in ref. 16, we obtain the scaling laws for the final volumes of the surface droplets after the solvent exchange: On the bottom wall it is

$$
V o l \sim \alpha^{3} h^{3}\left(\frac{c_{s, \text { wat }}}{\rho_{\text {oil }}}\right)^{3 / 2}\left(\frac{c_{s, \text { eth }}}{c_{s, \text { wat }}}-1\right)^{3 / 2} \mathrm{Pe}^{3 / 4}
$$

where $c_{s, \text { eth }}$ is the oil saturation concentration in ethanol, $c_{s, \text { wat }}$ is the oil saturation concentration in water, $h$ is the channel height, and $\rho_{\text {oil }}$ is the oil density. On the upper wall it is

$$
V o l \sim(1-\alpha)^{3} h^{3}\left(\frac{c_{s, \text { wat }}}{\rho_{\text {oil }}}\right)^{3 / 2}\left(\frac{c_{s, \text { eth }}}{c_{s, \text { wat }}}-1\right)^{3 / 2} \mathrm{Pe}^{3 / 4}
$$

On a vertical substrate with upward flow, the droplet volume is not influenced by the gravity, thus $\alpha=0.5$,

$$
V o l \sim \frac{1}{8} h^{3}\left(\frac{c_{s, \text { wat }}}{\rho_{\text {oil }}}\right)^{3 / 2}\left(\frac{c_{s, \mathrm{eth}}}{c_{s, \mathrm{wat}}}-1\right)^{3 / 2} \mathrm{Pe}^{3 / 4} .
$$

For the given channel height and Peclet number, the ratio of the droplet volume on the substrates placed at $0^{\circ}\left(V_{\text {lower }}\right), 90^{\circ}\left(V_{\text {vertical }}\right)$, and $180^{\circ}\left(V_{\text {upper }}\right)$ thus is

$$
V_{\text {lower }}: V_{\text {vertical }}: V_{\text {upper }}=8 \alpha^{3}: 1: 8(1-\alpha)^{3} \text {. }
$$


From our experimental results for the prefactors $(f(\theta))$ in Fig. 4b (channel height $h=$ $0.21 \mathrm{~mm}$, corresponding to $A r \approx 10$ ) we had the ratio $1: 2.5: 6.3$ or $0.4: 1: 2.52$, resulting in $\alpha \approx 0.37$ for the droplet volume ratio of the lower case to the vertical case, $\alpha \approx 0.32$ for the droplet volume ratio of the vertical case to the upper case, and $\alpha \approx 0.35$ for the droplet volume ratio of the lower case to the upper case. We conclude that $\alpha(A r \approx 10) \approx 0.35$ reasonably describes our data, which for this Ar seems to us to be a reasonable deviation from the value $\alpha(A r=0)=0.5$ without gravitational effects.

As stated already above, for small $A r \ll 1$, i.e., for small channel height $h$ (see the definition eq. (1), gravity effects will diminish. To confirm this, we conducted experiments in another horizontal channel with a smaller height of $h=0.07 \mathrm{~mm}$, corresponding to $A r \approx 0.35$, at flow rate $Q=400 \mu \mathrm{L} / \mathrm{min}$. The images and the size analysis of the droplets are shown in Fig. 6. Indeed, we found that there is no difference in the average volume of droplets on upper or lower walls, in contrast to the significant difference for the channel height $h=0.21 \mathrm{~mm}$. This result further supports the gravity effects on the droplet formation by the solvent exchange. Meanwhile, according to Eq. (4) for the situation without gravitational effect shown in Fig. 6b, the droplet volume reduces with the cube of the channel height. As the channel height decreases from $0.21 \mathrm{~mm}$ to $0.07 \mathrm{~mm}$, we obtain the droplet volume from $0.56 \mathrm{\mu m}^{3} / \mathrm{\mu m}^{2}$ to $0.03 \mathrm{\mu m}^{3} / \mathrm{\mu m}^{2}$. This is in good agreement with the prediction, given the resolution limit for the measurement of the channel height by an optical microscopy. The schematics of the flow profiles in the horizontal situation and the corresponding oil oversaturation pulses $\zeta(t)$ of the lower and upper wall for channel heights $0.21 \mathrm{~mm}(A r \approx 10)$ and $0.07 \mathrm{~mm}(A r \approx 0.35)$ are shown as Fig. $6 \mathrm{c}$ and $\mathrm{d}$, respectively. As the channel height decreased from $0.21 \mathrm{~mm}$ to $0.07 \mathrm{~mm}$ and Ar from $\gg 1$ to a value smaller than 1 , the value of $\alpha$ goes up from 0.35 to close to 0.5 , i.e., the value of the symmetric situation.

With our understanding of gravity effects, we now re-examine the experimental results of ref. 16, where the droplets were produced on the bottom wall of horizontal channels of three different heights. As the channel height increased from $h_{1}=0.33 \mathrm{~mm}, h_{2}=0.68 \mathrm{~mm}$ 


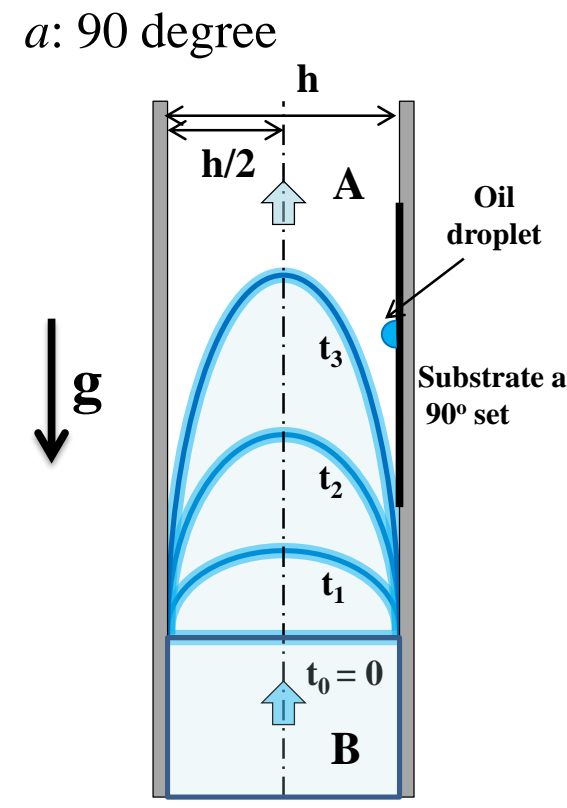

$b: 0$ and 180 degree

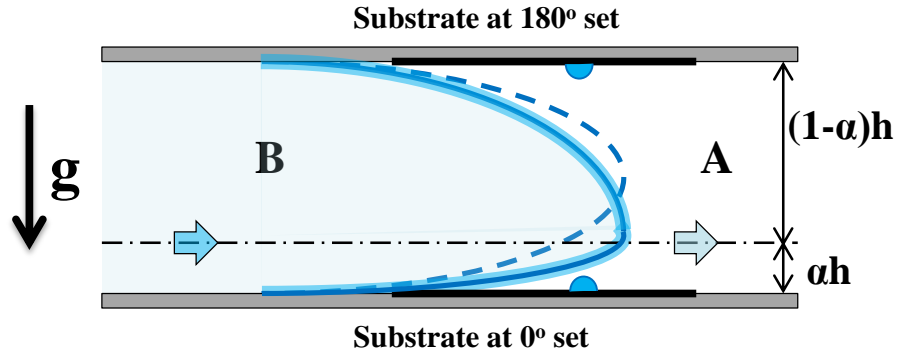

Figure 5: Geometrical schematics of solvent exchange process in different tilting angle $(a$ : $90^{\circ}, b: 0^{\circ}$ and $\left.180^{\circ}\right)$. The vector $g$ points in the direction of gravity. The dashed lines show the position of the maximal flow velocity at each situation.

to $h_{3}=2.21 \mathrm{~mm}$ (corresponding to Archimedes numbers of $A r_{1}=36, A r_{2}=314$, and $A r_{3}=10794$, respectively), the available time $\tau \sim h^{2} / D$ for the droplet growth increases. In ref. 16 it was also conjectured that in the two higher channels convection rolls further contribute to the better mixing, and hence enhance the droplet growth. The convection rolls occurred for the two higher channels as for those cases the Rayleigh number was above the critical value for onset of convection. In ref. 16 the ratio of the droplet volume was found to be approximate $1: 1.4: 2.8$ for the three channels of height $0.33 \mathrm{~mm}, 0.68 \mathrm{~mm}$, and $2.21 \mathrm{~mm}$, respectively. This ratio is much smaller than $h_{1}^{3}: h_{2}^{3}: h_{3}^{3}$, as according to equation (2) the gravity effects must be considered, too, namely (apart from the effects of the convection rolls) the volume ratio should be $\left(\alpha\left(A r_{1}\right) h_{1}\right)^{3}:\left(\alpha\left(A r_{2}\right) h_{2}\right)^{3}:\left(\alpha\left(A r_{3}\right) h_{3}\right)^{3}$, with a monotonously decreasing function $\alpha(A r)$. Clearly, the gravity effects reduce the droplet volume and thus partly compensate the collective contributions from the larger channel height and the convection.

As experimentally seen, gravity also influences the droplet formation in the vertical flow. 
(a) $\quad h=0.07 \mathrm{~mm} Q=400 \mu \mathrm{l} / \mathrm{min}$
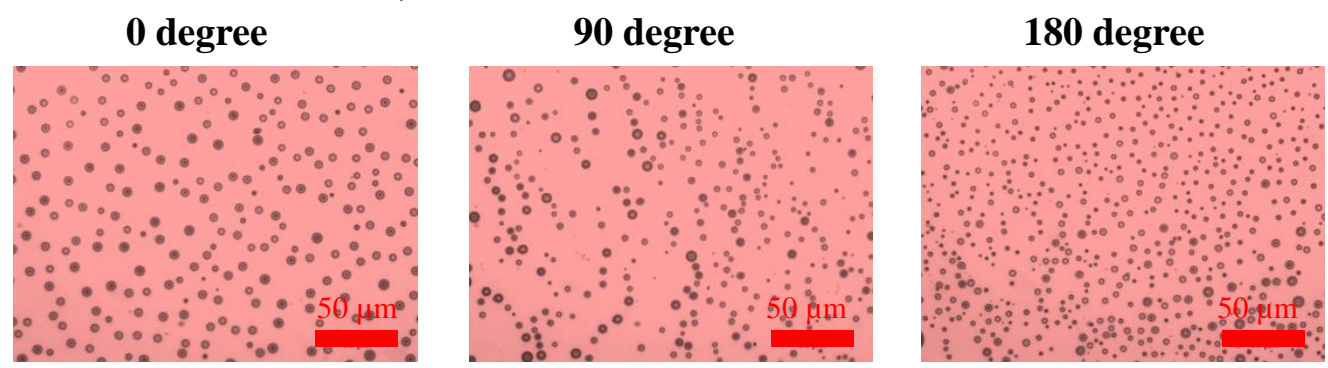

(b)

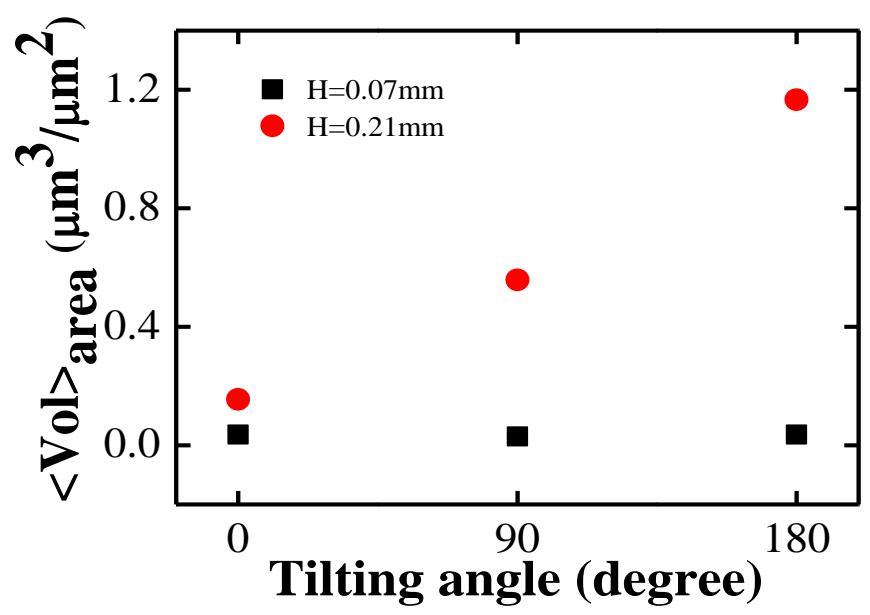

(c) $h=0.21 \mathrm{~mm}$
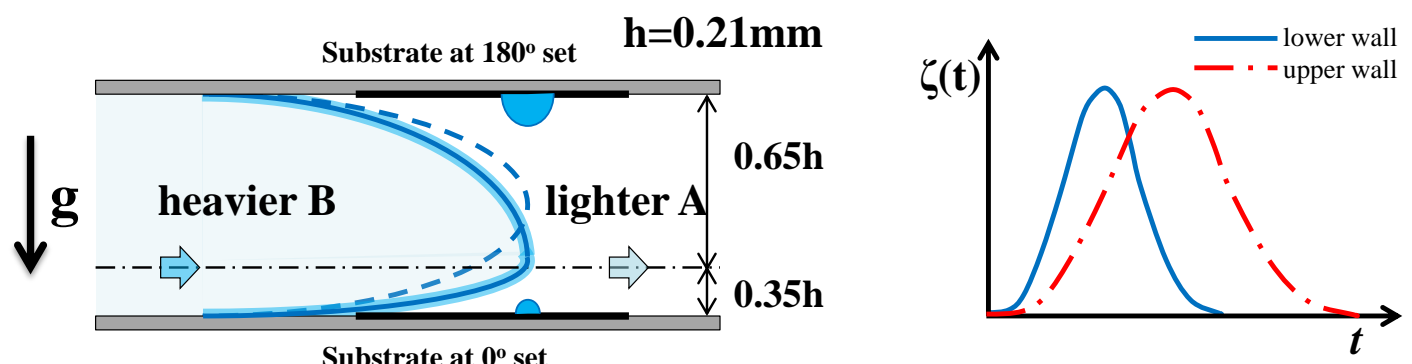

(d) $h=0.07 \mathrm{~mm}$
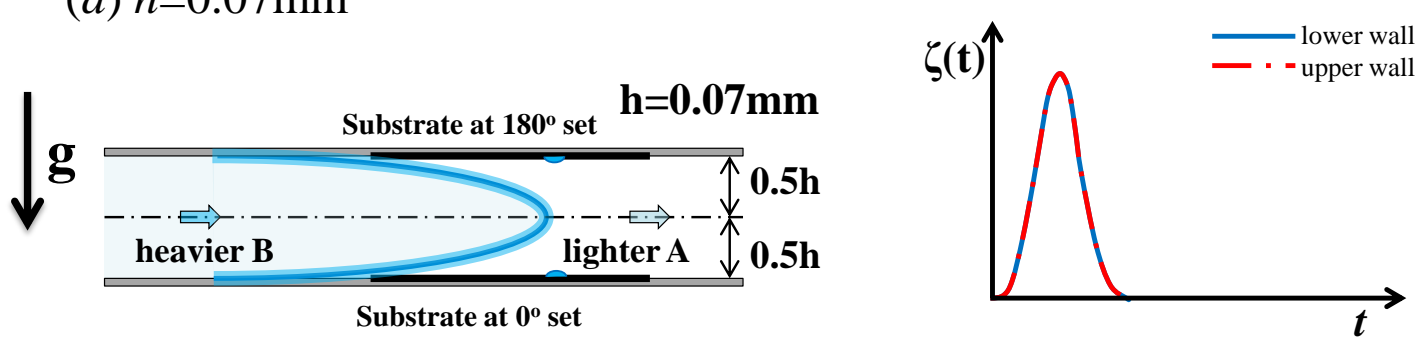

Figure 6: (a) Optical images and (b) average volume at $0^{\circ}, 90^{\circ}$ and $180^{\circ}$ tilting angles under the channel height $0.07 \mathrm{~mm}$ at the flow rate $400 \mu \mathrm{L} / \mathrm{min}$. (c) and $(d)$ Actual profile of flow at the horizontal set and the corresponding oil oversaturation $(\zeta(t))$ pulses of the lower and upper wall under the channel height $0.21 \mathrm{~mm}$ and $0.07 \mathrm{~mm}$, respectively. 
As seen from figures $2 \mathrm{c}$ and $3(\mathrm{a}-\mathrm{d})$, the droplet volume is larger for the downward flow than for the upward flow, which is particularly pronounced at slow flow rates. Two possible effects may contribute to this feature: First, although the flow rate of solution B was controlled, the body force due to the density difference $(\sim 10 \%)$ between solution A and B differently affects the fluid velocity for downward and upward flow: For downward flow, the actual flow rate may in fact be slightly larger due to gravity, leading to an effectively larger Peclet number. This effect would be particularly pertinent at low flow rates (as indeed seen in figure 4). Second, it could be that for downward flow (water pushing down the lighter ethanol) the Rayleigh-Taylor instability ${ }^{25,26}$ leads to enhanced mixing at the interface and thus to larger droplets. Again, this effect would be relatively more pronounced at low flow rates than at large flow rates, as then the instability has more time to develop. Additionally, we do not want to exclude that there are deficiencies in our control of the flow rate $Q$, in particular as the overall volume changes when mixing ethanol and water. We noticed that some droplets line up along the downward flow direction at slow flow rates, in contrast to the rather homogenous distribution over the entire surface in case of an upward flow. This suggests a non-uniform flow in our channel connected gravity effects.

\section{Conclusions}

In summary, we experimentally and theoretically investigate gravitational effect on the nucleation and growth of surface nanodroplets during the exchange of a lighter good solvent by a heavier poor solvent. With a channel height of $0.21 \mathrm{~mm}$, we found the droplet size is significantly larger on the upper wall than that on the lower wall. The gravitational effect is attributed to the non-symmetrical parabolic profile of the mixing front under gravity. Specifically, the position of the maximal flow velocity is shifted away from the upper wall towards the lower wall. Consequently, the diffusion time for the droplet growth is longer for the upper wall, and shorter for the lower wall. The ratio of the droplet volume on the 
lower and upper wall followed $\alpha^{3}:(1-\alpha)^{3}$, where the position of velocity peak $V_{\max }$ is $\alpha h$. When the channel is placed vertically in an upward flow, or is reduced in height, the gravity effect becomes negligible for the droplet formation. The results in this work have clearly demonstrated that the droplet nucleation and growth is coupled with the flow conditions near the boundary. The finding will guide the design of the flow conditions in the solvent exchange for the controlled formation of surface nanodroplets.

\section{Acknowledgement}

Acknowledgements: H.Yu acknowledges the Vice-Chancellor's PhD Scholarship in RMIT. D.L. gratefully acknowledges the support from European Research Council (ERC) and Netherlands Center for Multiscale Catalytic Energy Conversion (MCEC). X.H.Z. acknowledges the support from the Australian Research Council (FT120100473, DP140100805).

\section{References}

(1) Lohse, D.; Zhang, X. Surface nanobubbles and nanodroplets. Rev. Mod. Phys. 2015, $87,981-1035$.

(2) de Gennes, P.-G.; Brochard-Wyart, F.; Quéré, D. Capillarity and Wetting Phenomena; Springer New York: New York, NY, 2004.

(3) Widom, B. Capillarity and Wetting Phenomena: Drops, Bubbles, Pearls, Waves. Physics Today 2004, 57, 66-67.

(4) Vitale, S. A.; Katz, J. L. Liquid droplet dispersions formed by homogeneous liquidliquid nucleation: "The Ouzo effect". Langmuir 2003, 19, 4105-4110.

(5) Eslami, F.; Elliott, J. A. W. Stability analysis of microdrops during concentrating processes. J. Phys. Chem. B 2014, 118, 3630-41. 
(6) Belova, V.; Krasowsk, M.; Wang, D.; Ralston, J.; Shchukin, D. G.; Möhwald, H. Influence of adsorbed gas at liquid/solid iinterfaces on heterogeneous cavitation. Chem. Sci. 2013, 4, 248-256.

(7) Quéré, D. Wetting and Roughness. Annu. Rev. Mater. Res. 2008, 38, 71-99.

(8) Méndez-Vilas, A.; Jódar-Reyes, A. B.; González-Martín, M. L. Ultrasmall liquid droplets on solid surfaces: Production, imaging, and relevance for current wetting research. Small 2009, 5, 1366-1390.

(9) Meckenstock, R. U.; von Netzer, F.; Stumpp, C.; Lueders, T.; Himmelberg, A. M.; Hertkorn, N.; Schmitt-Kopplin, P.; Harir, M.; Hosein, R.; Haque, S.; SchulzeMakuch, D. Water droplets in oil are microhabitats for microbial life. Science 2014, 345, 673-676.

(10) Hong, J.; Edel, J. B.; DeMello, A. J. Micro- and nanofluidic systems for high-throughput biological screening. Drug Discovery Today 2009, 14, 134-146.

(11) Shemesh, J.; Ben Arye, T.; Avesar, J.; Kang, J. H.; Fine, A.; Super, M.; Meller, A.; Ingber, D. E.; Levenberg, S. Stationary nanoliter droplet array with a substrate of choice for single adherent/nonadherent cell incubation and analysis. Proc. Natl. Acad. Sci. U. S. A. 2014, 111, 11293-11298.

(12) Chiu, D. T.; Lorenz, R. M. Chemistry and biology in femtoliter and picoliter volume droplets. Acc. Chem. Res. 2009, 42, 649-658.

(13) Rissin, D. M.; Kan, C. W.; Campbell, T. G.; Howes, S. C.; Fournier, D. R.; Song, L.; Piech, T.; Patel, P. P.; Chang, L.; Rivnak, A. J.; Ferrell, E. P.; Randall, J. D.; Provuncher, G. K.; Walt, D. R.; Duffy, D. C. Single-molecule enzyme-linked immunosorbent assay detects serum proteins at subfemtomolar concentrations. Nat. Biotech. 2010, 28, 595-599. 
(14) Chen, Y.; Elshobaki, M.; Gebhardt, R.; Bergeson, S.; Noack, M.; Park, J.-M.; Hillier, A. C.; Ho, K.-M.; Biswas, R.; Chaudhary, S. Reducing optical losses in organic solar cells using microlens arrays: theoretical and experimental investigation of microlens dimensions. Phys. Chem. Chem. Phys. 2015, 17, 3723-30.

(15) Lou, S.-T.; Ouyang, Z.-Q.; Zhang, Y.; Li, X.-J.; Hu, J.; Li, M.-Q.; Yang, F.-J. Nanobubbles on solid surface imaged by atomic force microscopy. J. Vac. Sci. Technol., B 2000, 18, 2573-2575.

(16) Zhang, X.; Lu, Z.; Tan, H.; Bao, L.; He, Y.; Sun, C.; Lohse, D. Formation of surface nanodroplets under controlled flow conditions. Proc. Natl. Acad. Sci. U. S. A. 2015, 112, 9253-9257.

(17) Ma, A.; Xu, J.; Yu, L.; Zhang, X.; Wang, D.; Xu, H. Adsorbed emulsion droplets: capping agents for in situ heterogeneous engineering of particle surfaces. Chem. Commun. 2013, 49, 11563-11565.

(18) Zhang, X. H.; Ducker, W. Interfacial oil droplets. Langmuir 2008, 24, 110-115.

(19) Zhang, X. H.; Maeda, N.; Craig, V. S. J. Physical Properties of Nanobubbles on Hydrophobic Surfaces in Water and Aqueous Solutions. Langmuir 2006, 22, 5025-35.

(20) Hammond, B. R.; Stokes, R. H. Diffusion in binary liquid mixtures. Part 1.-Diffusion coefficients in the system ethanol + water at $25^{\circ}$. Trans. Faraday Soc. 1953, 49, 890895.

(21) Hills, E. E.; Abraham, M. H.; Hersey, A.; Bevan, C. D. Diffusion coefficients in ethanol and in water at 298 K: Linear free energy relationships. Fluid Phase Equilib. 2011, 303, $45-55$.

(22) Ermanyuk, E. V.; Gavrilov, N. V. Interaction of internal gravity current with an obstacle on the channel bottom. J. Appl. Mech. Tech. Phys. 2005, 46, 489-495. 
(23) Şal, S.; Gül, Ö. F.; Özdemir, M. The effect of sparger geometry on gas holdup and regime transition points in a bubble column equipped with perforated plate spargers. Chemical Engineering and Processing: Process Intensification 2013, 70, 259-266.

(24) Fabre, D.; Tchoufag, J.; Magnaudet, J. The steady oblique path of buoyancy-driven disks and spheres. J. Fluid Mech. 2012, 707, 24-36.

(25) Drazin, P.; Reid, W. H. Hydrodynamic stability; Cambridge University Press: Cambridge, 1981.

(26) Linden, P.; Redondo, J.; Youngs, D. Molecular mixing in Rayleigh-Taylor instability. Journal of Fluid Mechanics 1994, 265, 97-124. 


\section{Graphical TOC Entry}

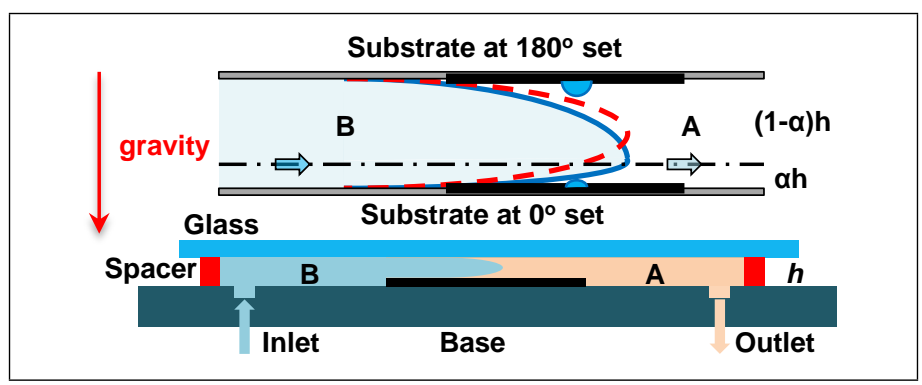

